\title{
Magnetic Reconnection and Extraplanar Diffuse Ionized Gas
}

\author{
Guido T. Birk ${ }^{1}$, Harald Lesch ${ }^{1}$ and Thomas Neukirch ${ }^{2}$
}

1 Institut für Astronomie und Astrophysik, LMU München, Scheinerstr. 1, 81679 München, Germany

2 School of Mathematical and Computational Sciences, University of St. Andrews, St. Andrews, Fife KY16 9SS, Scotland, United Kingdom

\begin{abstract}
Many observations indicate the occurrence of ionized gas in the distant halos of galaxies (among them our own). Since photoionization by stars (mainly O stars, young or evolved low mass stars depending on the kind of galaxy) does not seem to be exclusively responsible for the ionization of the hydrogen filaments that should otherwise cool and recombine quickly, the question arises which energy source may result in the quasistationary ionization. We propose that localized magnetic field dissipation in current filaments may contribute to the ionization of the extraplanar halo gas. The dissipated magnetic energy is converted into heat, which then is transferred to the neutrals, resulting in re-ionization on a time scale shorter than the recombination time scale. Quasistatic solutions are characterized by plasma temperatures and densities that agree nicely with the observed values for the diffuse ionized gas component of the Galactic interstellar medium.
\end{abstract}

\section{Introduction}

During the last decade it has become obvious that the existence of a diffuse ionized gas (DIG) component is of importance for our understanding of the interstellar medium. This component is widespread in the Galactic Halo, in the disk halo interface of spiral galaxies as well as in irregular, active and early type galaxies (recent reviews on the detection of DIG are given by Walterbos (1991) and Dettmar (1992)). The detection of the DIG component of the interstellar medium gives rise to the ionization problem: What are the energy sources that keep the extraplanar DIG ionized?

In this contribution we consider a fundamental plasma process that may help to solve the ionization problem: magnetic reconnection. Reconnection has proved to be an efficient heating process in quite different cosmical plasma regimes. Recently, it was shown, e.g. that this process can explain the X-ray emission of boundary regions of high-velocity clouds hitting the Galactic disk (Zimmer et al. 1997) and that it contributes to the small-scale solar coronal heating in bright points (Priest et al. 1993; Birk et al. 1997).

The train of thought is as follows: Extraplanar gas far away from Ostars cools and recombines effectively. In gas associations that are located in regions with inhomogeneous magnetic fields and localized electric current 
filaments, the recombination results in a lower thermal plasma pressure that counteracts the magnetic pressure in the central current regions and in a reduced electric conductivity mainly due to electron-neutral collisions. In this situation magnetic reconnection is likely to occur (e.g. Parker 1994). During this process, due to the local violation of ideal Ohm's law, magnetic field lines are reconnected and magnetic flux is dissipated. The free magnetic energy is mainly converted into heat via Ohmic dissipation and, consequently, the gas is re-heated and can be re-ionized.

\section{Quasistatic Stationary Heating and Ionization by Magnetic Dissipation}

Consider a mainly hydrogen quasi-neutral partially ionized plasma in ionization equilibrium in a quasistatic steady state. The relevant equations are the stationary ionization and energy balance equations

$$
\begin{gathered}
\alpha n_{p}^{2}=\iota n_{n} \\
\mathbf{j}^{2}=\sigma\left(L_{\text {brems }}^{\mathrm{rad}}+L_{\text {recom }}^{\mathrm{rad}}\right)
\end{gathered}
$$

where $n_{p}, n_{n}, \mathbf{j}, \alpha, \iota, \sigma, L_{\mathrm{brems}}^{\mathrm{rad}}$ and $L_{\text {recom }}^{\mathrm{rad}}$ denote the plasma (HII) and neutral gas (HI) particle densities, the current density, the recombination coefficient, the ionization frequency, the collisional conductivity due to electronneutral collisions and the radiative loss functions due to recombination radiation as well as bremsstrahlung (cf. Huba 1994 for the detailed expressions for the transport coefficients). The electric current filaments are assumed to be of the Harris sheet type (Harris 1962) for simplicity (we assume the magnetic field to vary in the $y$-direction):

$$
\mathbf{j}=7.7 \cdot 10^{-16}\left(\frac{B}{\mu \mathrm{G}}\right)\left(\frac{\delta}{\mathrm{pc}}\right)^{-1} \operatorname{sech}^{2}\left(\frac{y}{\delta}\right) \mathbf{e}_{z} \operatorname{statamp~cm^{-2}}
$$

where $\delta$ is the length scale of the inhomogeneity of the magnetic field, i.e. the half thickness of the current layer.

These equations can be rewritten as a set of non-linear algebraic equations for the plasma density $n_{p}$ and the electron temperature $T_{e}$ (measured in $e V$ ):

$$
\begin{gathered}
n_{p}^{3}+2.7 \cdot 10^{-23} \frac{B^{2}\left(n_{p}-n_{t}\right)}{L^{2} \cosh ^{4}(y / \delta)\left(1+E_{\infty} T_{e}^{-1 / 4}\right)}=0 \\
0.4+0.5 \ln \left(\frac{E_{\infty}}{T_{e}}\right)+0.5\left(\frac{T_{e}}{E_{\infty}}\right)^{1 / 3}=\frac{2 \cdot 10^{8} T_{e}\left(n_{t}-n_{p}\right) e^{-E_{\infty} / T_{e}}}{E_{\infty}^{5 / 2}\left(6+T_{e} E_{\infty}^{-1 / 4}\right) n_{p}}
\end{gathered}
$$

The ionization energy for hydrogen is $E_{\infty}=13.6 \mathrm{eV}$ and the lower estimate for the magnetic field strength is about $5 \mu \mathrm{G}$ from radio data (Beck et al. 
1996). The total particle density $n_{t}$ and $\delta$ are the only free input parameters of this idealized model. Fig. 1 shows for example the spatial profiles of the electron temperature $T_{e}$, plasma density $n_{p}$ and neutral gas density $n_{n}$ for $n_{t}=1 \mathrm{~cm}^{-3}$ and $\delta=10^{-11} \mathrm{pc}$. The electron temperature is close to the observed value of the HII temperature. The electron and ion temperatures should not differ significantly since electron-ion collisions lead to a relatively fast (as compared to the recombination time scale) thermalization. The plasma density has its maximum in the central reconnection region (i.e. the central region of the current filament) and decreases with increasing distance from the current sheet. The ionization rate is $60 \%$ which agrees quite well with observed values in some Galactic DIG regions. We note that
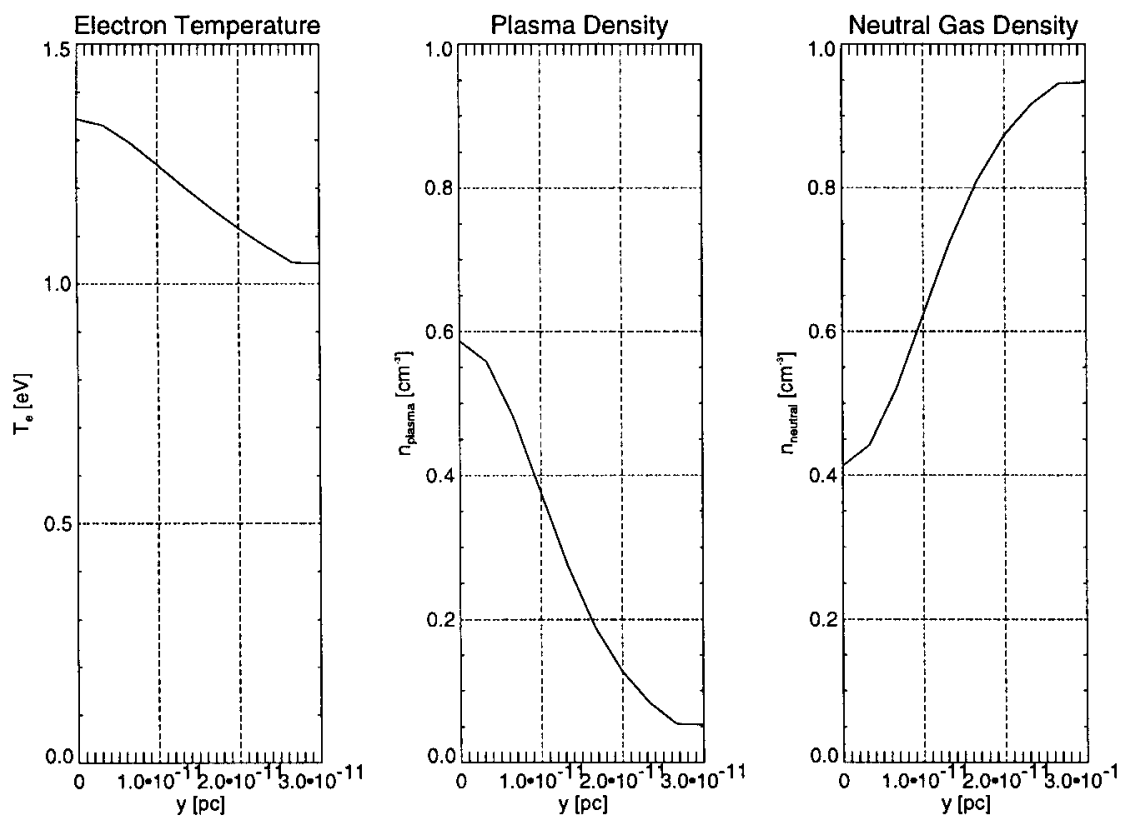

Fig. 1. Spatial profiles of the electron temperature, the plasma and the neutral gas density for a total densities of $n_{t}=1 \mathrm{~cm}^{-3}$

at least for the relatively wide parameter range studied, $n_{t}=0.15 . .20 \mathrm{~cm}^{-3}$ and $\delta=10^{-11} . .10^{-8} \mathrm{pc}$, we find reasonable values for the HII densities and temperatures. 


\section{Stationary Heating by Magnetic Reconnection}

Dropping the assumption of a static configuration we are dealing with stationary magnetic reconnection (e.g. Parker 1994). In the framework of this description the magnetic diffusivity gives rise to dynamical dissipation of the inflowing magnetic field as rapidly as magnetic flux is convected in. The time scale of the dissipation process depends on the width of the current filament $\Delta$, and can be estimated (cf. Parker 1994) as $\tau_{\text {rec }}=5 \cdot 10^{4} \mathrm{~s}$ for $\Delta$ comparable to the thickness of the filament $\delta\left(\delta=10^{9} \mathrm{~cm}\right.$ obtained from the condition of stationarity) up to $\tau_{\text {rec }}=10^{12} \mathrm{~s}$ for the slowest case $(\Delta=\delta S$ where $S$ is the magnetic Reynolds number) which is still faster than the recombination time scale $t_{\text {rec }}=10^{13} \mathrm{~s}$.

The rate of conversion of magnetic energy caused by magnetic reconnection at maximum has to satisfy (cf. Dettmar 1992):

$$
\frac{d}{d t} \int \frac{B^{2}}{8 \pi} d^{3} r \approx 10^{-12} \frac{V}{\tau_{\text {rec }}} \mathrm{G}^{2} \approx 10^{42} \mathrm{ergs}^{-1}
$$

which implies a quite small (as compared to the extension of the Galactic halo) volume $V \approx 10 \mathrm{pc}^{3}$ in which a sufficient amount of magnetic energy can be dissipated in many individual reconnection processes for $\Delta \approx \delta$.

We note that the refreshment rate of magnetic fields (caused by galactic fountains, supernovae, Galactic dynamo etc.) has to be taken into account in order to put quantitative restrictions on our model.

Acknowledgements. This work was supported by the DFG through the grant ME 745/18-1 and by the United Kingdom Particle Physics and Astronomy Research Council.

\section{References}

Beck, R., Brandenburg, A., Moss, D. et al. (1996): ARAA 34, 155-206

Birk, G.T., Dreher, J., Neukirch, T. (1997): Magnetic Reconnection in the Solar Atmosphere, eds. R. D. Bentley and J. T. Mariska, ASP Conf. Ser. 111, 89-94

Dettmar, R.-J. (1992): Fund. Cosm. Phys. 15, 143-208

Harris, E.G. (1962): Nuovo Cim. 23, 115

Huba, J.D. (1994): NRL Plasma Formulary (Naval Research Laboratory, Washington), 39-57

Parker, E.N. (1994): Spontaneous Current Sheets in Magnetic Fields (Oxford University Press), 291-300

Priest, E.R., Parnell, C.E., Martin, S.F. (1993): ApJ 427, 459-474

Walterbos, R.A.M. (1991): The Interstellar Disk-Halo Connection in Galaxies, ed. H. Bloemen, IAU Symp. 144, 223-236

Zimmer, F., Lesch, H., Birk, G.T. (1997): A\&A 320, 746-756 